BMJ Open Sport \& Exercise Medicine

\section{Effectiveness of high-intensity interval training for weight loss in adults with obesity: a randomised controlled non- inferiority trial}

To cite: D'Amuri A, Sanz JM, Capatti $\mathrm{E}$, et al. Effectiveness of high-intensity interval training for weight loss in adults with obesity: a randomised controlled noninferiority trial. BMJ Open Sport \& Exercise Medicine 2021;7:e01021. doi:10.1136/ bmjsem-2020-001021

- Additional supplemental material is published online only. To view, please visit the journal online (http://dx. doi. org/10.1136/bmjsem-2020 001021).

$A D$ and JMS are joint first authors.

Accepted 5 July 2021

Check for updates

(c) Author(s) (or their employer(s)) 2021. Re-use permitted under CC BY-NC. No commercial re-use. See rights and permissions. Published by BMJ.

${ }^{1}$ Department of Translational Medicine, University of Ferrara, Ferrara, Emilia Romagna, Italy ${ }^{2}$ Department of Chemical and Pharmaceutical Sciences, University of Ferrara, Ferrara, Emilia Romagna, Italy ${ }^{3}$ Medical Department, University Hospital of Ferrara Arcispedale Sant'Anna, Ferrara, Emilia Romagna, Italy

${ }^{4}$ Department of Medical

Sciences, University of Udine, Udine, Friuli Venezia Giulia, Italy

Correspondence to Professor Angelina Passaro; psn@unife.it

\section{ABSTRACT}

Introduction Obesity treatment guidelines suggest moderate-intensity continuous training (MICT), but the patient's compliance to this indication remains low. Highintensity interval training (HIIT) is a time sparing training mode whose metabolic effects are not clear. This study aimed to determine whether a 12-week HIIT was more effective than MICT for weight loss in obese adults.

Methods 44 obese subjects were randomised and trained with isoenergetic treadmill exercises for 12 weeks: MICT ( $60 \%$ of maximal oxygen peak, V ${ }_{2}$ peak) or HIIT (3-7 repetition of $3 \mathrm{~min} 100 \%$ of $\mathrm{VO}_{2}$ peak interspersed by 1.5 min $50 \%$ of $\mathrm{V}_{2}$ peak). The primary outcome was a change in body weight; the secondary outcomes were changes in body composition, blood pressure, lipid profile, glycaemia, insulin and V0 peak.

Results 32 subjects ( $53 \%$ male, mean age: 38.5 years, mean body mass index: $35.5 \mathrm{~kg} / \mathrm{m}^{2}$ ) completed the trial. MICT and HIIT showed comparable effect within groups in weight loss $(-6.0 \mathrm{~kg}(-9.0 \mathrm{~kg}$ to $-3.0 \mathrm{~kg}) \mathrm{vs}-5.7 \mathrm{~kg}(-8.3$ $\mathrm{kg}$ to $-3.1 \mathrm{~kg}))$, changes in fat mass $(-2.9 \%(-4.4 \%$ to $-1.4 \%)$ vs $-3.6 \%(-5.9 \%$ to $-1.2 \%))$, fat free mass $(-5.3 \%$ $(-7.8 \%$ to $-2.8 \%)$ vs $-5.5 \%(-8.3 \%$ to $-2.6 \%))$, diastolic blood pressure $(-5.5 \mathrm{~mm} \mathrm{Hg}(-10.6 \mathrm{~mm} \mathrm{Hg}$ to $-0.3 \mathrm{~mm}$ $\mathrm{Hg}$ ) vs $-5.8 \mathrm{~mm} \mathrm{Hg}(-11.3 \mathrm{~mm} \mathrm{Hg}$ to $-0.3 \mathrm{~mm} \mathrm{Hg})$ ) and low-density lipoprotein cholesterol $(-16.4 \mathrm{mg} / \mathrm{dL}(-30.8 \mathrm{mg} /$ $\mathrm{dL}$ to $-2.0 \mathrm{mg} / \mathrm{dL})$ vs $-14.7 \mathrm{mg} / \mathrm{dL}(-25.6 \mathrm{mg} / \mathrm{dL}$ to -3.8 $\mathrm{mg} / \mathrm{dL})$ ). There was a significant change between groups in VO peak (HIIT: +461.6 mL (329.3-593.8 mL); MICT: +170.5 $\mathrm{mL}^{2}(86.7-254.4 \mathrm{~mL}) ; \mathrm{p}<0001$ ) and duration of sessions (HIIT: $35.0 \mathrm{~min}$ (31.7-35.6 min); MICT: $46.5 \mathrm{~min}$ (40.2-48.3 min); $\mathrm{p}<0.001)$. No significant changes in systolic blood pressure, high-density lipoprotein cholesterol, triglycerides, glycaemia or plasma insulin were observed.

Conclusions In healthy adults with obesity, HIIT compared with MICT induced similar weight loss and cardiovascular risk factors improvement but resulted in a larger increase in cardiorespiratory fitness over a shorter period.

\section{INTRODUCTION}

Obesity is a major contributor to poor health in most countries ${ }^{1}$ because it is a risk factor for cardiovascular and metabolic disorders ${ }^{2}$ and

\section{Key messages}

What is already known

- Physical exercise, in obesity treatment, is fundamental to maintain a negative energy balance, facilitate weight loss and increase cardiorespiratory fitness (CRF).

- Guidelines suggest that moderate-intensity continuous training (MICT) should be considered the preferable exercise mode.

- Promising data suggest that high-intensity interval training (HIIT), a time sparing exercise mode, could be at least as effective as MICT. Studies conducted, so far, are not conclusive as populations analysed are small and heterogeneous.

What are the new findings

- HIIT is as effective as MICT in inducing weight loss despite requiring shorter training sessions, with comparable side effects and dropout rate.

- HIIT is superior to MICT in improving CRF, a parameter strongly associated with a cardiovascular disease (CVD) risk reduction.

- HIIT represents an effective tool to increase compliance of obese patients to change their lifestyle.

- Clinicians should consider HIIT protocols as part of a strategy for obesity treatment and primary cardiovascular prevention, especially in obese adults, with minimal comorbidities.

increases the risk of all-cause mortality. ${ }^{3}$ Lifestyle modification is a cornerstone of obesity treatment ${ }^{4}$ resulting in better outcomes than pharmacological intervention. ${ }^{5}$ The beneficial effects of physical activity on weight control and central adiposity reduction are well documented. ${ }^{6}$ Although restriction of energy intake is more efficient than exercise to achieve weight loss, exercise is more efficient in reducing visceral adiposity ${ }^{6}$ and maintaining lean body mass. ${ }^{7}$ Moreover, exercise may confer benefits beyond weight loss by increasing cardiorespiratory fitness $(\mathrm{CRF}) .{ }^{8} \quad$ Several guidelines suggest that 
aerobic training should be the first-line exercise mode for obese individuals. ${ }^{9}{ }^{10}$ Training intensity, defined as moderate (ranging from $46 \%$ to $63 \%$ of maximal oxygen uptake $\left(\mathrm{VO}_{2} \max \right)$ and high (ranging from $64 \%$ to $90 \%$ $\left.\mathrm{VO}_{2} \max \right),{ }^{11}{ }^{2}$ identifies different types of aerobic exercises. Historically, moderate-intensity continuous training (MICT) is the most studied in obesity treatment because of its higher rate of fat oxidation ${ }^{12}$ and lower risk of injury ${ }^{13}$ in this range of intensity. Unfortunately, training at moderate intensity requires high volume activity to reach relevant volumes of energy expenditure, resulting in weight loss. Guidelines suggest at least 225-420 min of MICT to obtain clinically significant weight loss $(\geq 5 \%)$ without dietary restriction. ${ }^{9}$ This could represent one of the main obstacles to therapeutic adherence since the lack of time is a common reason limiting exercise. ${ }^{14}$ For this reason, high-intensity interval training (HIIT) has recently become a popular strategy in weight loss programmes in the general population. ${ }^{15}$ HIIT is defined by short bouts of high-intensity exercise, alternating with periods of low-intensity exercise. HIIT allows to accomplish equicaloric training sessions in a significantly shorter period (up to $40 \%$ less time) and seems to be at least non-inferior to MICT in terms of changes in body weight ${ }^{16}$ and even superior in reducing body fat. ${ }^{17}$ Moreover, HIIT induces greater improvements in $\mathrm{CRF}^{18}$ and cardiometabolic disorders. ${ }^{19}$ A recent meta-analysis shows that HIIT is as effective as MICT in inducing weight loss and body composition modification ${ }^{16}$ and superior in increasing CRF. ${ }^{20}$ However, this meta-analysis has some limitations: the number of subjects enrolled in the studies is low and populations are heterogeneous. Therefore, larger samples are required to understand the effects of different exercises modalities better.

The primary aim of this study was to evaluate, in healthy obese adults, whether a 12-week HIIT programme was not inferior to MICT in terms of weight loss. We evaluated changes in the waist and hip circumferences, body composition, arterial blood pressure, lipid profile, glucose metabolism and CRF as secondary outcomes.

\section{MATERIAL AND METHODS Study protocol \\ Study design}

This is a randomised, single-blind, single-centre, parallelgroup study that compares 12 weeks of HIIT and MICT in obese healthy adults. Subjects were randomly allocated with a 1:1 ratio into two arms through a computerised random number generator. The trial adhered to established procedures to maintain separation between staff that deliver the intervention and staff that take outcome measurements. Research assistants who delivered the intervention did not take outcome measurements, staff members who obtained outcome measurements and dieticians were not informed of the training group assignment. Both groups underwent a nutritional evaluation by a dietician, and all subjects were given a tailored hypocaloric diet to follow. As a non-inferiority trial, the study's primary aim was to demonstrate that HIIT is as effective as MICT in terms of weight loss in obesity management. We also evaluated changes in the waist and hip circumferences, body composition, arterial blood pressure, lipid profile, glucose metabolism and CRF as secondary outcomes.

All measurements and samples were obtained at baseline data collection (BDC) and post-training data collection (PTDC). Anthropometrics indexes and physical capacities were monitored monthly to adjust diet calories requirement and programme of physical activity.

\section{Participants}

Inclusion criteria were age between 18 years old and 50 years old and body mass index (BMI) between $30 \mathrm{~kg}$ / $\mathrm{m}^{2}$ and $55 \mathrm{~kg} / \mathrm{m}^{2}$. Exclusion criteria were the previous participation in weight management programmes, being physically active, a history of cardiovascular, respiratory, neurologic, muscularskeletal, psychiatric and endocrine diseases, active treatment with any drugs known to influence energy metabolism. Written informed consent was obtained, and the ethics committee of the Friuli-VeneziaGiulia Region approved the study. A physical activity questionnaire (International Physical Activity Questionnaire - Short Form (IPAQ-SF)) was administered to exclude potential volunteers who were physically active (defined as subjects doing any continuous activity $>20$ min more than once a week, indicative of a moderate physical activity level).$^{21}$ The study took place at the Exercise Physiology Laboratory of the University of Udine, Italy, between September 2017 and June 2018.

Among 60 volunteers evaluated, 44 subjects considered eligible for the study were enrolled and equally randomised into the arms of the study (HITT vs MICT). Among non-eligible subjects, three did not meet BMI criteria, two had a psychiatric disease, two had cardiovascular disease, one had polycystic ovary syndrome, one was affected by muscularskeletal illness, one had a history of binge drinking disorder, two could not guarantee adherence to the information provided and four were assuming beta-blockers.

After randomisation, only 32 subjects (16 in the HIIT arm and 16 in the MICT arm) completed the study, and their data were used in subsequent analysis. Twelve subjects dropped out: seven (four in the HIIT arm and three in the MICT arm) did not show adequate adherence to the workouts and five (two in the HIIT arm and three in the MICT arm) withdrew their consent (figure 1).

\section{Physical exercise}

Subjects underwent a 12-week programme of physical exercise consisting of 3 sessions/week of MICT or HIIT. Training sessions took place under the supervision of a research assistant to ensure that each subject completed at least $90 \%$ of the exercise sessions. Assuming that weight loss depends only on an energy deficit, regardless of training mode or intensity, we chose training regimes to maximise the improvements of $\mathrm{VO}_{2} \max$ as suggested 


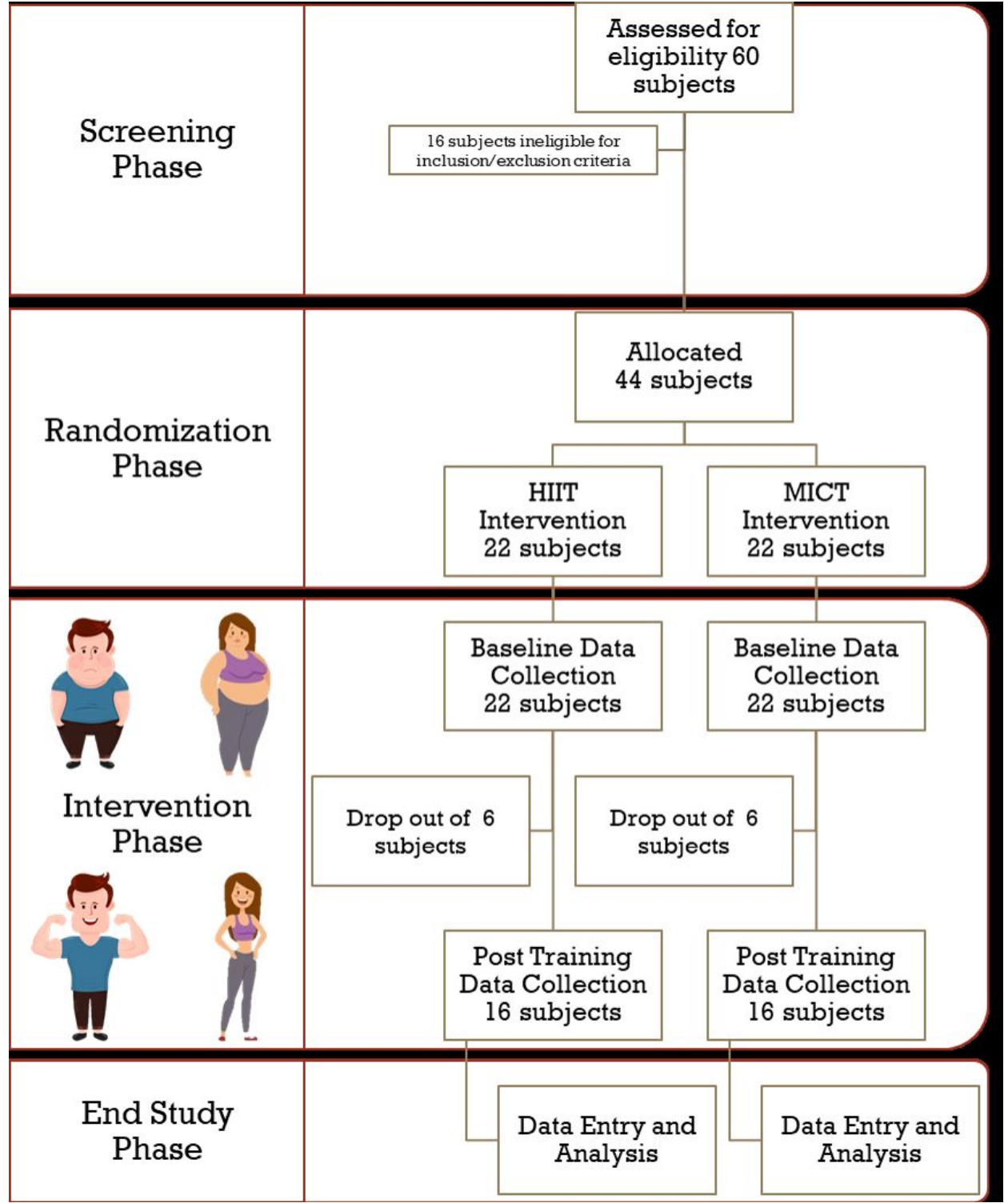

Figure 1 Flowchart of the overall study population. HIIT, high-intensity interval training; MICT, moderate-intensity continuous training.

by Buchheit $e t a l^{22}$, keeping the intensity close to $100 \%$ of the velocity correspondent to peak oxygen uptake $\left(\mathrm{VO}_{2}\right.$ peak) and about $50 \%$ during recovery intervals; furthermore, they suggest to use HIIT with long interval (few minutes) or short interval $(<1 \mathrm{~min})$ with a work/ recovery ratio less than 1 . We have chosen a protocol with 3-minute intervals because it is more feasible on a treadmill than shorter intervals.

The intensity of MICT on the treadmill was set at an heart rate (HR) corresponding to $60 \%$ of $\mathrm{BDC}-\mathrm{VO}_{2}$ peak. HIIT consisted of 3-7 repetition of $3 \mathrm{~min}$ at $100 \%$ $\mathrm{VO}_{2}$ peak interspersed by $1.5 \mathrm{~min}$ at $50 \% \mathrm{VO}_{2}$ peak. The intensity was set up, adjusting the slope of the treadmill as observed during the incremental test. The intensity was progressively increased by gradually increasing the speed up to a maximum of $5.5 \mathrm{~km} /$ hour and then gradually increasing the slope. Both HIIT than MICT training sessions included 10 min of warm-up $\left(50 \% \mathrm{VO}_{2}\right.$ peak $)$ and
5 min of cool-down ( $50 \% \mathrm{VO}_{2}$ peak). Participants wore a HR monitor during training to ensure the correct intensity was prescribed. The duration of the training sessions was planned to obtain equal amounts of energy expenditure in the two groups: $20 \mathrm{~kJ}(4.78 \mathrm{kcal})$ per $\mathrm{kg}$ of fat-free mass (FFM). At the end of each month, aerobic tests were performed to assess physical capacities and adjust physical training intensity individually.

\section{Diet and nutritional education}

Every subject underwent a nutritional evaluation by a specialised dietician. Participants fill out a food diary of 4 days, at the beginning and before PTDC, to evaluate meal habits, nutrients and energy intake. According to Italian recommended dietary allowances at BDC, each participant received a personalised hypocaloric diet. ${ }^{23}$ There was a run-in period of 6 weeks during which energy supply was about 1.3 times the initial basal metabolic rate, 
estimated using the Harris-Benedict equation. After this period, subjects underwent a further energy deficit of $500 \mathrm{kcal} /$ day for the last 6 weeks.

\section{Outcome measures}

Anthropometric characteristics, body composition and blood pressure

Body composition was measured by bioelectrical impedance analysis (BIA; Human IM Plus, DS Dietosystem, Milan, Italy), according to Lukaski et $a l^{24}$. FM and FFM were calculated with equations derived from people of different ages and grades of obesity (fat-specific formulae) by using a two-compartment model. ${ }^{25} \mathrm{~A}$ trained operator measured blood pressure with a sphygmomanometer following current guidelines. ${ }^{26}$

Physical capacities, training measures and adverse events $\mathrm{VO}_{2}$ peak was determined using a graded exercise test on a motorised treadmill $(\mathrm{H} / \mathrm{P} /$ Cosmos Sports \& Medical GmBh, Germany) under medical supervision. Subjects avoided strenuous exercise the day before the test and came to the laboratory after 12-hour fasting. Each test was undertaken simultaneously, in different periods, for the same subject and comprised a 5-minute rest period followed by walking in stages of 4-minute duration. Speed in $\mathrm{m} / \mathrm{s}$ and incline in $\%$ followed a sequence: $1.11 \mathrm{~m} / \mathrm{s}$ (0\%), $1.11 \mathrm{~m} / \mathrm{s}(3 \%), 1.39 \mathrm{~m} / \mathrm{s}(3 \%), 1.39 \mathrm{~m} / \mathrm{s}(6 \%)$, $1.53 \mathrm{~m} / \mathrm{s}(6 \%), 1.53 \mathrm{~m} / \mathrm{s}(9 \%), 1.53 \mathrm{~m} / \mathrm{s}(12 \%), 1.53 \mathrm{~m} / \mathrm{s}$ (13\%), $1.53 \mathrm{~m} / \mathrm{s}(15 \%), 1.53 \mathrm{~m} / \mathrm{s}(18 \%), 1.53 \mathrm{~m} / \mathrm{s}(21 \%)$ and $1.53 \mathrm{~m} / \mathrm{s}(24 \%)$. During the experiment, ventilatory and gas exchange responses were measured continuously by indirect calorimetry (CPET, Cosmed, Italy). The system's flow metre and gas analyser were calibrated using, respectively, a $3 \mathrm{~L}$ calibration syringe and calibration gas $\left(16.00 \% \mathrm{O}_{2} ; 4.00 \% \mathrm{CO}_{2}\right)$. An ECG was recorded continuously and displayed online for visual monitoring during the exercise test, and HR was measured with a dedicated monitor device (Garmin, USA). The $\mathrm{VO}_{2}$ peak was estimated for each subject considering the last $20 \mathrm{~s}$ of the graded exercise tests.

The amount of training session, duration of any session and energy expenditure during exercise were recorded. Any adverse or unexpected events were recorded.

\section{Nutritional habits}

Participants completed a 1-week food diary to estimate energy intake before randomisation, after diet prescription and at the end of the study.

\section{Biologic samples and measures}

After overnight fasting, blood samples were collected from each subject and centrifuged in the absence or presence of EDTA to obtain serum and plasma. Highdensity lipoprotein cholesterol (HDL-C) was measured after precipitation of apoproteins B (apo B) containing lipoproteins ${ }^{27}$; total cholesterol (Tot-C) and triglycerides levels were assayed in serum by the Trinder method. The coefficient of variation was $<2 \%$ for Tot-C and HDL-C and $<5 \%$ for triglycerides for intra-batch and inter-batch, respectively. Friedewald's formula calculated low-density lipoprotein cholesterol (LDL-C) plasma levels. Plasma glucose (PG) was measured using standard enzymatic methods (FAR S.R.L., Italy). The coefficient of variation was $<3 \%$ for intra-assay. Fasting insulin levels were assayed using an ultrasensitive insulin ELISA kit (Mercodia AB, Sweden). The coefficient of variation was $<3 \%$ for interassay. Insulin resistance was estimated with homeostasis model assessment (HOMA-IR).

\section{Statistical analysis}

To prove non-inferiority of HIIT in respect to MICT, standard treatment, in inducing weight loss of 5\% in 3 months (one-sided significance level: 0.05 , target power: 0.8 , allocation ratio: $1: 1, \mathrm{SD}$ assumed: \pm 1.15 ), it was calculated a sample size of 16 subjects per arm; a dropout rate of $20 \%$ was supposed, so the target was to recruit at least 40 participants. Primary and secondary outcomes were evaluated in all patients that completed the trial. Waist and hip circumference analysis was performed by sex. Variables were expressed as mean \pm SD or median (MD) and 95\% CIs; categorical variables were expressed as frequencies. The normal distribution of continuous variables was assessed using Kolmogorov-Smirnov and Shapiro-Wilk tests. Variables not normally distributed were log-transformed before entering the statistical analysis. At BDC, differences between groups and variable of interest were analysed with variance analysis (ANOVA), with Bonferroni for post-hoc analysis and $\chi^{2}$ test for categorical variables. At the same time, MDs were compared by non-parametric tests (Kruskal-Wallis). Correlations between continuous variables were tested with a Pearson's correlation test for variables with a normal distribution. In contrast, variables with non-normal distribution were analysed after $\log$ transformation or with a non-parametric test (Spearman's test). The data are described with the correlation coefficient $(r)$ and the significance level against the null hypothesis ( $p$ value). Variations between BDC and PTDC of the variables of interest were analysed by t-test for repeated measures and general linear model (GLM) for repeated measures, within-subjects and between-subjects tests. Statistical analysis was performed using SPSS V.26.0 software, and statistical significance was set at a $p$ value $<0.05$. When significant, a practical effect was also reported.

\section{RESULTS}

Subjects in the two arms of the study did not significantly differ at baseline (table 1). Among enrolled subjects, 32 participants completed the study (16 HIIT and 16 MICT), with a dropout rate of $27 \%$ (figure 1).

\section{Primary outcome}

After 12 weeks of training, weight decreased in both arms of the study. HIIT decreased body weight by -5.7 $\mathrm{kg}((-8.3 \mathrm{~kg}$ to $-3.1 \mathrm{~kg}) ; \mathrm{p}$ value $=0.001)$ while MICT by $-6.0 \mathrm{~kg}((-9.0 \mathrm{~kg}$ to $-3.0 \mathrm{~kg}) ; \mathrm{p}<0001)$ without significant differences between the experimental groups. This 
Table 1 Description of subjects by treatment group (MICT vs HIIT) at baseline data collection

\begin{tabular}{llll} 
& MICT & HIIT & \\
\cline { 2 - 2 } Variable & Number & Number & P value \\
\hline Subjects & 16 & 16 & \\
Sex, males/females & $9 / 7$ & $8 / 8$ & 0.723 \\
\hline Smoker, never/in the past/current & $12 / 4 / 0$ & $9 / 4 / 1$ & 0.553 \\
\hline Hypertension medications, no/yes & $16 / 0$ & $16 / 0$ & \\
\hline Hyperlipidaemia medications, no/yes & $16 / 0$ & $16 / 0$ & \\
\hline Diabetes medications, no/yes & $16 / 0$ & $16 / 0$ & 0.335 \\
\hline Age $($ years $)$ & Mean $\pm S D$ & Mean $\pm S D$ & 0.479 \\
\hline Weight $(\mathrm{kg})$ & $37 \pm 9$ & $40 \pm 7$ & 0.514 \\
\hline $\mathrm{BMI}^{2}\left(\mathrm{~kg} / \mathrm{m}^{2}\right)$ & $107.1 \pm 17.4$ & $103.4 \pm 10.9$ & 0.632 \\
$\mathrm{VO}_{2}$ peak $(\mathrm{mL})$ & $36.1 \pm 5.1$ & $35.1 \pm 3.6$ & 0.824 \\
$\mathrm{VO}_{2}$ peak $/$ weight $(\mathrm{mL} / \mathrm{kg})$ & $3016.3 \pm 782.7$ & $2888.7 \pm 706.2$ & \\
\hline
\end{tabular}

BMI, body mass index; HIIT, high-intensity interval training; MICT, moderate-intensity continuous training; $\mathrm{VO}_{2}$ peak, peak oxygen uptake.

implies a reduction in BMI of $-1.9 \mathrm{~kg} / \mathrm{m}^{2}\left(\left(-2.7 \mathrm{~kg} / \mathrm{m}^{2}\right.\right.$ to $\left.\left.-1.0 \mathrm{~kg} / \mathrm{m}^{2}\right) ; \mathrm{p}=0.001\right)$ in HIIT and $-2.1 \mathrm{~kg} / \mathrm{m}^{2}((-3.2$ $\mathrm{kg} / \mathrm{m}^{2}$ to $\left.\left.-1.1 \mathrm{~kg} / \mathrm{m}^{2}\right) ; \mathrm{p}<0.001\right)$ in MICT with no difference between groups (table 2).

\section{Secondary outcome}

Anthropometric characteristics and body composition

Waist circumference was reduced within groups both in males $(-11.4 \mathrm{~cm}(-15.6 \mathrm{~cm}$ to $-7.1 \mathrm{~cm}) ; \mathrm{p}<0.001)$ and females $(-17.8 \mathrm{~cm}(-22.8 \mathrm{~cm}$ to $-12.8 \mathrm{~cm}) ; \mathrm{p}<0.001)$ with a greater reduction in males trained with HIIT $(-8.5$ $\mathrm{cm}(-10.9 \mathrm{~cm}$ to $-6.1 \mathrm{~cm}))$ compared with MICT $(-1.8$ $\mathrm{cm}(-6.1 \mathrm{~cm}$ to $2.5 \mathrm{~cm}))(\mathrm{p}=0.039)$; on the contrary, no difference between groups was observed in females. Hip circumference was reduced within groups both in males $(-3.0 \mathrm{~cm}(-4.8 \mathrm{~cm}$ to $-1.2 \mathrm{~cm}) ; \mathrm{p}=0.003)$ and females $(-6.6 \mathrm{~cm}(-9.6 \mathrm{~cm}$ to $-3.6 \mathrm{~cm}) ; \mathrm{p}<0.001)$ with no difference between groups (table 2).

Both absolute and percentage FM was reduced within groups $(-5.4 \mathrm{~kg}(-7.2 \mathrm{~kg}$ to $-3.6 \mathrm{~kg})$ and $-3.2 \%(-4.6 \%$ to $-1.9 \%) ; \mathrm{p}=0.001$ for both) with no difference between groups. Absolute FFM did not change, within groups, while an increase in FFM was observed (3.3\% (1.9\% to $4.7 \%) ; \mathrm{p}=0.001)$ ), with no difference between groups (table 2).

Blood pressure, lipid profile and glucose metabolism

No changes in systolic blood pressure (SBP) were recorded. We observed a within groups reduction in diastolic blood pressure (DBP) $(-5,6(-9,2--2,0) \mathrm{P}$-value $0,003)$, which did not differ between training modes. Resting HR decreased after intervention within groups $-10,7(-13,9--7,5)$ bpm; $p$ value $<0001))$ without any influence of the type of exercise (table 3 ).

Tot-C and LDL-C decreased within groups $(-17.8 \mathrm{mg} /$ $\mathrm{dL}(-27.9 \mathrm{mg} / \mathrm{dL}$ to $-7.79 \mathrm{mg} / \mathrm{dL})$ and $-15.6 \mathrm{mg} / \mathrm{dL}$
$(-24.3 \mathrm{mg} / \mathrm{dL}$ to $-6.8 \mathrm{mg} / \mathrm{dL})$, respectively; $\mathrm{p}=0.001$ for both), with no difference between groups; HDL-C did not change after intervention. Triglycerides, PG, insulin and HOMA-IR did not change after intervention (table 3). No HDL-C and total cholesterol (TC)/HDL-C ratio decreased after intervention within groups $(-16.0$ $\mathrm{mg} / \mathrm{dL}(-25.0 \mathrm{mg} / \mathrm{dL}$ to $-7.1 \mathrm{mg} / \mathrm{dL}) ; \mathrm{p}=0.001 ;-0.223$ ( -0.453 to 0.001$) ; \mathrm{p}=0.05$, respectively), with no difference between groups. Similarly, apo-B100 was reduced significantly within groups $(-7.4 \mathrm{mg} / \mathrm{dL}(-11.9 \mathrm{mg} / \mathrm{dL}$ to $-2.9 \mathrm{mg} / \mathrm{dL}$ ); $\mathrm{p}=0.002$ ) with no difference between groups. Apo-A1 did not change after intervention (data not shown).

\section{Cardiorespiratory fitness}

Mean $\mathrm{VO}_{2}$ peak increased compared with baseline within groups $(316.1 \mathrm{~mL}(241.0-3911 \mathrm{~mL}) ; \mathrm{p}<0.001))$ with a greater increase in HIIT $(461.6 \mathrm{~mL}(329.3-593.8 \mathrm{~mL}))$ than in MICT $(170.5 \mathrm{~mL}(86.7-254.4 \mathrm{~mL}))(\mathrm{p}<0.001)$. $\mathrm{VO}_{2}$ peak/weight augmented after training within groups $(4.9 \mathrm{~mL} / \mathrm{kg}(3.9-5.9 \mathrm{~mL} / \mathrm{kg}) ; \mathrm{p}<0.001))$ with a greater increase in HIIT $(6.5 \mathrm{~mL} / \mathrm{kg}(4.9-8.1 \mathrm{~mL} / \mathrm{kg}))$ than MICT $(3.3 \mathrm{~mL} / \mathrm{kg}(2.0-4.6 \mathrm{~mL} / \mathrm{kg}) ; \mathrm{p}=0.003)$ (table 4$)$.

\section{Training measures and adverse events}

The MD number of training sessions executed was $35 / 37$ (32-37) for MICT and 36/37 (32-37) for HIIT ( $\mathrm{p}=0.812$ ); the MD duration of exercise session was 46.5 min (40.2$48.3 \mathrm{~min})$ in MICT and $35.0 \mathrm{~min}(31.7-35.6 \mathrm{~min})$ in HIIT $(-24 \%$; $\mathrm{p}<0.001)$. The MD energy expenditure for session was $394.4 \mathrm{kcal}(338.3-441.4 \mathrm{kcal})$ for MICT and $332.0 \mathrm{kcal}(313.4-386.5 \mathrm{kcal})$ for HIIT $(\mathrm{p}=0.189)$. No adverse events occurred in both groups (online supplemental table 1). 


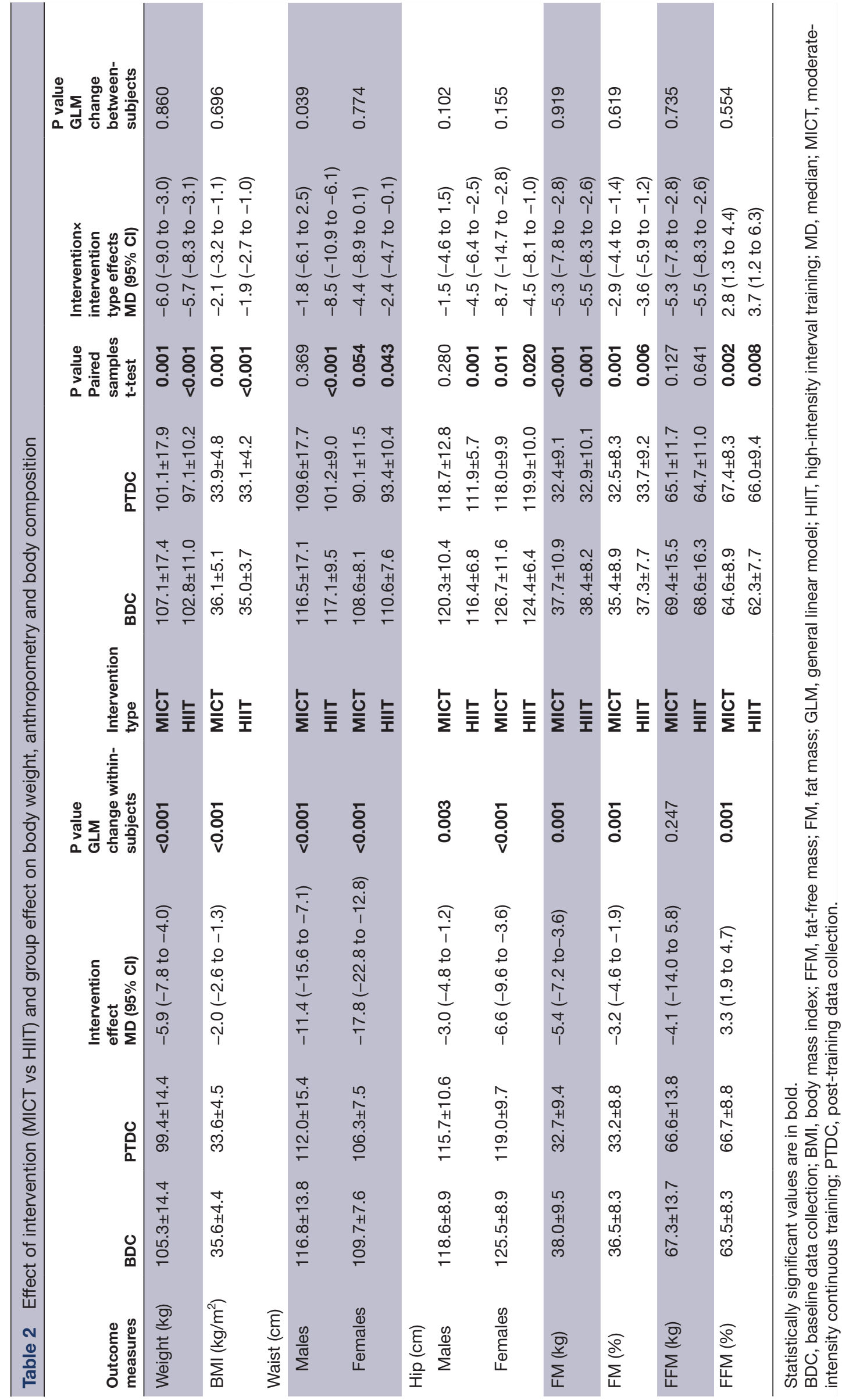




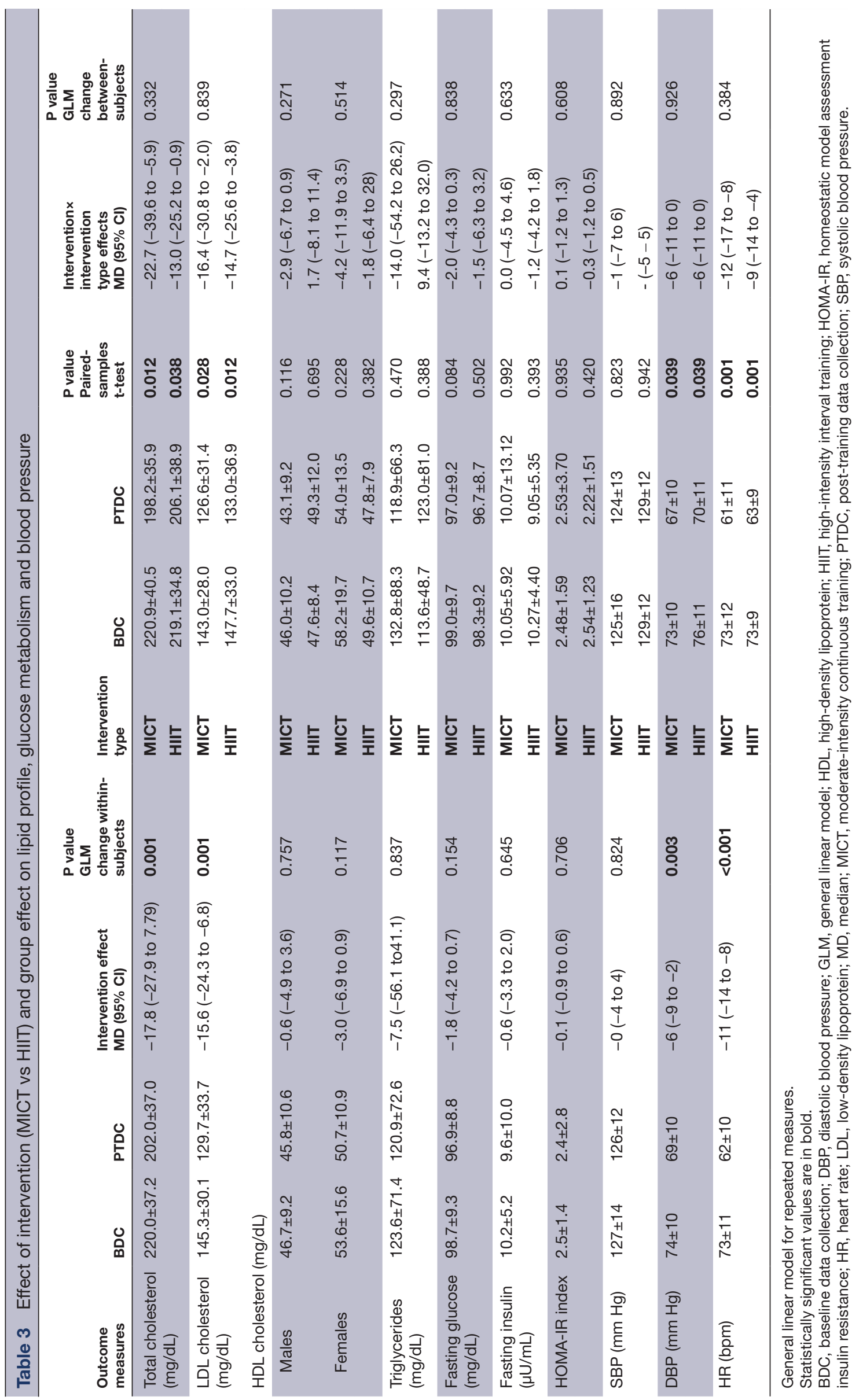


Nutritional habits

There was no difference between meal calories prescribed and calorie intakes deducted by food diaries, both in the run-in phase and in the last 6 weeks of intervention, with no difference between groups (table 4 ).

\section{DISCUSSION}

In this interventional trial of 12 weeks of training, HIIT appears to be non-inferior to MICT in inducing weight loss in obese, otherwise healthy, adults.

After 12 weeks of intervention, subjects in the HIIT group achieved a significant weight loss and BMI reduction, which was approximately $6.1 \%$ of initial body weight. Similar results were observed in the MICT group, a trend fully following guidelines indications. ${ }^{28}$ This was associated with a positive modification of body composition, such as reducing FM and an increase in FFM. These results follow previous observations, ${ }^{16}$ showing that different training modes have a similar impact on weight and fat mass loss. Interestingly, although both exercise protocols led to a reduction in waist and hip circumference, HIIT was able to induce a greater reduction of waist circumference in males, suggesting it could represent a more favourable strategy to treat visceral obesity. Similarly, Zhang et $a l^{29}$ observed that HIIT could be more effective than MICT in reducing visceral adipose tissue when evaluated by the gold-standard technique in a cohort of obese young females.

In this study, the intervention reduced DBP but not SBP. This contrasts with the known effect of exercise in healthy adults, generally resulting in a reduction of both DBP and SBP. ${ }^{30}$ However, our finding aligns with what was observed in obese people who start training without a dietary intervention. ${ }^{31}$ Similar results emerged from meta-analysis, evaluating HIIT protocols lasting less than 12 weeks, but not for those lasting longer. ${ }^{19}$ In this study, the effect of HIIT was similar to MICT in reducing blood pressure, as previously observed.$^{32}$ Also, resting $\mathrm{HR}$ was reduced after the training intervention, as expected. ${ }^{33}$ Both MICT $^{33}$ and HIIT $^{34}$ have been demonstrated to reduce HR effectively. However, to the best of our knowledge, this is the first study to compare these two exercise modes directly, demonstrating that they have similar efficacy in reducing HR in obese adults. On the lipid metabolism side, exercise led to a significant improvement of lipid profile, with a reduction of Tot-C, LDL-C (-15.6 mg/dL), non-HDL cholesterol $(-16.0 \mathrm{mg} /$ $\mathrm{dL}$ ) and apoB-100 (data not shown). This is in line with previous observations demonstrating the positive effect of training on lipoprotein metabolism, ${ }^{31}$ regardless of exercise modality. ${ }^{35}$ This supports the idea that the effect of exercise on lipid metabolism is more related to exercise-induced energy expenditure than to exercise intensity. ${ }^{36}$ Surprisingly, in our study, we did not observe any modification in HDL-C nor triglycerides concentrations, despite the known benefits of exercise. ${ }^{31}$ Similarly, we did not observe any significant modification of PG, insulin and HOMA-IR, as described by other authors. ${ }^{37}$ 
The obese subjects we enrolled in were otherwise healthy, and the prevalence of metabolic syndrome was low. Moreover, the kind of intervention we chose in our study is not a long-term one. We speculate that metabolic ameliorations following training are less evident in subjects who have not developed metabolic abnormalities related to obesity or requires a longer intervention.

Although the effect of exercise might appear modest on subclinical or biochemical parameters, like blood pressure or lipid profile ${ }^{38}$ its role on strong clinical outcomes is well established. ${ }^{39}$ This effect is probably mediated by the improvement in $\mathrm{CRF}^{39}$ a surrogate of whole-body physiological function, predicting outcomes better than the sum of test investigating the function of different organs or singular risk factors. As previously described, ${ }^{40}$ in this study, CRF increased in both arms by an amount comparable to what has already been described ${ }^{18}$ with a greater improvement in HIIT, showing a 23\% and nearly 2 METs increase, compared with $10.4 \%$ and less than 1 metabolic equivalents (METs) increase after MICT. Such an increment has to be considered clinically important, given that 1 estimated MET increase in CRF has been associated with $13 \%$ and $15 \%$ reductions in all-cause and cardiovascular diseases/coronary artery disease (CVD/ CHD) mortality, respectively. ${ }^{41}$ These numbers appear even greater in primary prevention, a field in which no other approach has been shown as effective as a lifestyle intervention. Moreover, there is emerging evidence that high levels of CRF may attenuate or even eliminate the elevated CVD and all-cause mortality risk in overweight and obese individuals. ${ }^{42}$ As previously reported, ${ }^{16}$ HIIT requires shorter sessions than MICT to burn the same amount of calories. Moreover, it is known that despite HIIT contains high intensity and more tiring phases, it is generally at least as appreciated as MICT. ${ }^{43}$ We observed similar dropout rates in the HIIT and MICT arms, without significant adverse events, as previously reported in other studies. ${ }^{44}$

This study has some limitations. First, the sample size was tailored to detect differences in the primary outcome and, therefore, the trial is underpowered to show eventual sex difference in training mode effects. Similarly, this trial is underpowered to detect significant differences in CRF; thus, we are aware that this finding, although positive, should be interpreted with caution. Body composition was assessed by BIA, a technique less accurate than gold-standard methods and unable to detect small differences in body composition. Moreover, an intervention protocol lasting 12 weeks could be too short to produce a metabolic effect and not long enough to estimate the long-term compliance to exercise of obese subjects. In addition, participants in our study were obese, and inferences should not be made concerning other populations.

The study's strengths are the feasibility of the training modes that many obese adults could execute without supervision, and the contemporary assessment of all the main cardiovascular risk factors (blood pressure, PG, lipid profile, weight and CRF). So, this protocol could be reliably and effectively delivered in general clinical practice as part of a strategy for obesity treatment and primary cardiovascular prevention. In conclusion, HIIT is comparable to MICT in inducing weight loss and improving major cardiovascular risk factors. HIIT may be more effective in increasing CRF over the same time compared with MICT in obese adult people. Moreover, HIIT requires less time than MICT to execute an isocaloric exercise. Considering all these features, HIIT may be an alternative treatment tool in obese adults with minimal comorbidities who report lack of time as their main barrier to MICT.

Acknowledgements We would like to thank the participants in the study for their time and effort to ensure the success of the project. We thank the nursing staff at the Department of Public Health, A.A.S. n. 3 "Alto Friuli- Collinare-Medio Friuli", Gemona del Friuli, Udine, Italy, for their qualified assistance during the study. Results of the study are presented clearly, honestly and without fabrication, falsification or inappropriate data manipulation.

Contributors Our work is a product of the intellectual environment of the whole team. All members have contributed in various degrees to its birth. Conception and design of the study: AP, AD and SL. Acquisition of data: JMS, AP, AD, EC, FDV and FV. Data analysis and interpretation, and drafting of the article: AP and AD. Critical revision of the article: JMS, EC, FDV, FV, SL, EDN and GZ. All authors read and approved the final manuscript.

Funding The study was supported by the University of Udine and University of Ferrara research funds.

Competing interests None declared.

Patient and public involvement Patients and/or the public were not involved in the design, or conduct, or reporting, or dissemination plans of this research.

Patient consent for publication Not required.

Ethics approval The present study was approved by the National Ethical Committee of the Friuli-Venezia-Giulia Region (protocol number: 1764). The purposes and objectives of this study were carefully explained to the participants, and written informed consent was obtained. Data are available through the corresponding author upon request and for justified reasons.

Provenance and peer review Not commissioned; internally peer reviewed. Data availability statement Data are available upon reasonable request.

Open access This is an open access article distributed in accordance with the Creative Commons Attribution Non Commercial (CC BY-NC 4.0) license, which permits others to distribute, remix, adapt, build upon this work non-commercially, and license their derivative works on different terms, provided the original work is properly cited, appropriate credit is given, any changes made indicated, and the use is non-commercial. See: http://creativecommons.org/licenses/by-nc/4.0/.

\section{ORCID iD}

Angelina Passaro http://orcid.org/0000-0001-8462-7000

\section{REFERENCES}

1 Di Cesare M, Bentham J, Stevens GA, et al. Trends in adult bodymass index in 200 countries from 1975 to 2014: a pooled analysis of 1698 population-based measurement studies with $19 \cdot 2$ million participants. Lancet 2016;387:1377-96.

2 Poirier P, Giles TD, Bray GA, et al. Obesity and cardiovascular disease: pathophysiology, evaluation, and effect of weight loss. Circulation 2006;113:898-918.

3 Flegal KM, Kit BK, Orpana $\mathrm{H}$, et al. Association of all-cause mortality with overweight and obesity using standard body mass index categories: a systematic review and meta-analysis. JAMA 2013;309:71.

4 Jakicic JM, Clark K, Coleman E, et al. American College of sports medicine position stand. appropriate intervention strategies for weight loss and prevention of weight regain for adults. Med Sci Sports Exerc 2001;33:2145-56.

5 Knowler WC, Barrett-Connor E, Fowler SE, et al. Reduction in the incidence of type 2 diabetes with lifestyle intervention or metformin. N Engl J Med 2002;346:393-403. 
6 Verheggen RJHM, Maessen MFH, Green DJ, et al. A systematic review and meta-analysis on the effects of exercise training versus hypocaloric diet: distinct effects on body weight and visceral adipose tissue. Obes Rev 2016;17:664-90.

7 Petridou A, Siopi A, Mougios V. Exercise in the management of obesity. Metabolism 2019;92:163-9.

8 Davidson LE, Hunt SC, Adams TD. Fitness versus adiposity in cardiovascular disease risk. Eur J Clin Nutr 2019;73:225-30.

9 Donnelly JE, Blair SN, Jakicic JM, et al. American College of sports medicine position stand. appropriate physical activity intervention strategies for weight loss and prevention of weight regain for adults. Med Sci Sports Exerc 2009;41:459-71.

10 Fogelholm M, Stallknecht B, Van Baak M. ECSS position statement: exercise and obesity. Eur J Sport Sci 2006;6:15-24.

11 Garber CE, Blissmer B, Deschenes MR, et al. Quantity and quality of exercise for developing and maintaining cardiorespiratory, musculoskeletal, and neuromotor fitness in apparently healthy adults. Med Sci Sport Exerc 2011;43:1334-59.

12 Achten J, Jeukendrup AE. Optimizing fat oxidation through exercise and diet. Nutrition 2004;20:716-27.

13 Balady GJ, Chaitman B, Driscoll D, et al. Recommendations for cardiovascular screening, staffing, and emergency policies at health/ fitness facilities. Circulation 1998:97:2283-93.

14 Trost SG, Owen N, Bauman AE. Correlates of adults' participation in physical activity: review and update. / Etude des facteurs sociaux, personnels et culturels entourant la participation a une activite physique pour des adultes. Med Sci Sport Exerc 2002;34:1996-2001.

15 Obert J, Pearlman M, Obert L, et al. Popular weight loss strategies: a review of four weight loss techniques. Curr Gastroenterol Rep 2017:19:61.

16 Wewege M, van den Berg R, Ward RE, et al. The effects of highintensity interval training vs. moderate-intensity continuous training on body composition in overweight and obese adults: a systematic review and meta-analysis. Obes Rev 2017;18:635-46.

17 Türk Y, Theel W, Kasteleyn MJ, et al. High intensity training in obesity: a meta-analysis. Obes Sci Pract 2017;3:258-71.

18 Milanović Z, Sporiš G, Weston M. Effectiveness of high-intensity interval training (HIT) and continuous endurance training for VO2max improvements: a systematic review and meta-analysis of controlled trials. Sports Med 2015;45:1469-81.

19 Batacan RB, Duncan MJ, Dalbo VJ, et al. Effects of high-intensity interval training on cardiometabolic health: a systematic review and meta-analysis of intervention studies. Br J Sports Med 2017:51:494-503.

$20 \mathrm{Su}$ L, Fu J, Sun S, et al. Effects of HIIT and MICT on cardiovascular risk factors in adults with overweight and/or obesity: a metaanalysis. PLoS One 2019;14:e0210644.

21 Craig CL, Marshall AL, Sjöström M, et al. International physical activity questionnaire: 12-country reliability and validity. Med Sci Sports Exerc 2003;35:1381-95

22 Buchheit M, Laursen PB, Training H-II. Solutions to the programming puzzle. Sport Med 2013;43:313-38.

23 laia M. Standard italiani per la cura dell'obesit. Quad ACP 2012;19:207.

24 Lukaski HC, Bolonchuk WW, Hall CB, et al. Validation of tetrapolar bioelectrical impedance method to assess human body composition. J Appl Physiol 1986;60:1327-32.

25 Gray DS, Bray GA, Gemayel N, et al. Effect of obesity on bioelectrical impedance. Am J Clin Nutr 1989;50:255-60.

26 Williams B, Mancia G, Spiering W. Practice guidelines for the management of arterial hypertension of the European Society of cardiology and the European Society of hypertension. Blood Press 2018;2018:314-40.

27 Burstein M, Scholnick HR, Morfin R. Rapid method for the isolation of lipoproteins from human serum by precipitation with polyanions. $J$ Lipid Res 1970;11:583-95.

28 Durrer Schutz D, Busetto L, Dicker D, et al. European practical and patient-centred guidelines for adult obesity management in primary care. Obes Facts 2019;12:40-66.

29 Zhang H, Tong TK, Qiu W, et al. Comparable effects of high-intensity interval training and prolonged continuous exercise training on abdominal visceral fat reduction in obese young women. $J$ Diabetes Res 2017;2017:1-9.

30 Cornelissen VA, Smart NA. Exercise training for blood pressure: a systematic review and meta-analysis. J Am Heart Assoc 2013;2: 0004473.

31 Shaw K, Gennat H, O'Rourke P, et al. Exercise for overweight or obesity. Cochrane Database Syst Rev 2006:CD003817.

32 Costa EC, Hay JL, Kehler DS, et al. Effects of high-intensity interval training versus moderate-intensity continuous training on blood pressure in adults with pre- to established hypertension: a systematic review and meta-analysis of randomized trials. Sports Med 2018;48:2127-42

33 Cao L, Li X, Yan P, et al. The effectiveness of aerobic exercise for hypertensive population: a systematic review and meta-analysis. $J$ Clin Hypertens 2019;21:868-76.

34 Rodrigues JAL, Ferrari GD, Trapé Átila Alexandre, et al. $\beta$ adrenergic interaction and cardiac autonomic function: effects of aerobic training in overweight/obese individuals. Eur J Appl Physiol 2020;120:613-24.

35 Wood G, Murrell A, van der Touw T, et al. HIIT is not superior to MICT in altering blood lipids: a systematic review and meta-analysis. BMJ Open Sport Exerc Med 2019;5:e000647.

36 Kraus WE, Houmard JA, Duscha BD, et al. Effects of the amount and intensity of exercise on plasma lipoproteins. N Engl J Med 2002;347:1483-92.

37 Elmer DJ, Laird RH, Barberio MD, et al. Inflammatory, lipid, and body composition responses to interval training or moderate aerobic training. Eur J Appl Physiol 2016;116:601-9.

38 Lavie CJ, Kachur S, Sui X. Impact of fitness and changes in fitness on lipids and survival. Prog Cardiovasc Dis 2019;62:431-5.

39 Lavie CJ, Ozemek C, Carbone S, et al. Sedentary behavior, exercise, and cardiovascular health. Circ Res 2019;124:799-815.

40 Ramos JS, Dalleck LC, Tjonna AE, et al. The impact of high-intensity interval training versus moderate-intensity continuous training on vascular function: a systematic review and meta-analysis. Sports Med 2015;45:679-92.

41 Kodama S, Saito K, Tanaka S, et al. Cardiorespiratory fitness as a quantitative predictor of all-cause mortality and cardiovascular events in healthy men and women: a meta-analysis. JAMA 2009;301:301.

42 Kennedy AB, Lavie CJ, Blair SN. Fitness or fatness. JAMA 2018;319:231.

43 Oliveira BRR, Santos TM, Kilpatrick M, et al. Affective and enjoyment responses in high intensity interval training and continuous training: a systematic review and meta-analysis. PLoS One 2018;13:e0197124.

44 Vella CA, Taylor K, Drummer D. High-intensity interval and moderate-intensity continuous training elicit similar enjoyment and adherence levels in overweight and obese adults. Eur J Sport Sci 2017;17:1203-11. 